\title{
The 12-Word Philadelphia Verbal Learning Test Performances in Older Adults: Brain MRI and Cerebrospinal Fluid Correlates and Regression-Based Normative Data
}

\author{
Katherine A. Gifford ${ }^{a} \quad$ Dandan Liu $^{b}$ Jacquelyn E. Neal ${ }^{b}$ \\ Michelle A. Babicz ${ }^{a}$ c Jennifer L. Thompson ${ }^{a}$ Lily E. Walljasper ${ }^{a}$ \\ Margaret E. Wiggins ${ }^{e}$ Maxim Turchan $^{a}$ Kimberly R. Pechman ${ }^{a}$ \\ Katie E. Osborn ${ }^{a}$ Lealani Mae Y. Acosta ${ }^{a}$ Susan P. Bell ${ }^{a} d$ \\ Timothy J. Hohman ${ }^{a}$ David J. Libon ${ }^{f}$ Kaj Blennow ${ }^{g}$ h \\ Henrik Zetterberg ${ }^{g-j} \quad$ Angela L. Jefferson ${ }^{a}$ \\ a Vanderbilt Memory and Alzheimer's Center, Department of Neurology, Vanderbilt \\ University Medical Center, Nashville, TN, USA; ${ }^{b}$ Department of Biostatistics, Vanderbilt \\ University Medical Center, Nashville, TN, USA; ' ${ }^{\circ}$ Department of Psychology, University \\ of Houston, Houston, TX, USA; d Divisions of Cardiovascular and Geriatric Medicine, \\ Department of Medicine, Vanderbilt University Medical Center, Nashville, TN, USA; \\ e Department of Clinical and Health Psychology, University of Florida, Gainesville, \\ FL, USA; ${ }^{f}$ Departments of Geriatrics and Gerontology and Psychology, New Jersey Institute \\ for Successful Aging, School of Osteopathic Medicine, Rowan University, Stratford, \\ NJ, USA; ${ }^{9}$ Department of Psychiatry and Neurochemistry, Institute of Neuroscience and \\ Physiology, The Sahlgrenska Academy at University of Gothenburg, Mölndal, Sweden; \\ ${ }^{h}$ Clinical Neurochemistry Laboratory, Sahlgrenska University Hospital, Mölndal, Sweden; \\ i Department of Molecular Neuroscience, UCL Institute of Neurology, London, UK; \\ jUK Dementia Research Institute at UCL, London, UK
}

\section{Keywords}

Aging · Alzheimer's disease · Biomarkers · Episodic memory · Psychometrics · Normative data Structural neuroimaging $\cdot$ Temporal lobe $\cdot$ White matter hyperintensities .

Cerebrospinal fluid

\section{Abstract}

Background/Aims: This study evaluated neuroimaging and biological correlates, psychometric properties, and regression-based normative data of the 12-word Philadelphia Verbal Learning Test (PVLT), a list-learning test. Methods: Vanderbilt Memory and Aging Project participants free of clinical dementia and stroke ( $n=230$, aged $73 \pm 7$ years) completed a neuropsychological protocol and brain MRI. A subset $(n=111)$ underwent lumbar puncture for 
analysis of Alzheimer's disease (AD) and axonal integrity cerebrospinal fluid (CSF) biomarkers. Regression models related PVLT indices to MRI and CSF biomarkers adjusting for age, sex, race/ethnicity, education, $A P O E-\varepsilon 4$ carrier status, cognitive status, and intracranial volume (MRI models). Secondary analyses were restricted to participants with normal cognition (NC; $n=127$ ), from which regression-based normative data were generated. Results: Lower PVLT performances were associated with smaller medial temporal lobe volumes $(p<0.05)$ and higher CSF tau concentrations $(p<0.04)$. Among NC, PVLT indices were associated with white matter hyperintensities on MRI and an axonal injury biomarker (CSF neurofilament light; $p<$ 0.03). Conclusion: The PVLT appears sensitive to markers of neurodegeneration, including temporal regions affected by AD. Conversely, in cognitively normal older adults, PVLT performance seems to relate to white matter disease and axonal injury, perhaps reflecting non-AD pathways to cognitive change. Enhanced normative data enrich the clinical utility of this tool.

(C) 2018 The Author(s)

Published by S. Karger AG, Basel

\section{Introduction}

Alzheimer's disease (AD) is an important public health issue, especially as the population continues to age. Episodic memory impairment is an essential and early feature of AD [1, 2]. Patterns of performance obtained from verbal list-learning tasks assessing episodic memory provide a wealth of information regarding underlying brain-behavior relations. Moreover, episodic memory impairment is one of the best predictors of future AD conversion [1]. Verbal list-learning tasks are widely recognized as critical tools for early detection of AD given their link with early pathological changes of AD [3]. Specifically, these measures correspond to amyloid deposition as measured by cerebrospinal fluid (CSF) amyloid-beta $42\left(\mathrm{~A} \beta_{42}\right)$ [4] and PET radioligands [5], neurodegeneration evidenced by hippocampal atrophy [6] and CSF tau [4], and white matter disease captured by white matter hyperintensities (WMH) [7].

The Philadelphia Verbal Learning Test (PVLT) is a serial list-learning task that was modeled after the California Verbal Learning Test (CVLT) [8]. It was originally developed as a 9-word list to assess episodic learning and memory in older adults with dementia for the purposes of differential diagnosis $[9,10]$. More recently, the PVLT has been updated to include a 12-word version for use in detecting early stages of cognitive impairment in older adults [11]. Similar to the CVLT, a multi-item shopping list consisting of three categories is presented to increase ecological validity with a familiar, real-life activity. The PVLT measures rate of acquisition, retrieval (free and cued recall), recognition (encoding), and susceptibility to proactive and retroactive interference. After five free recall trials of the List A shopping list, a distractor shopping list (List B) is presented with semantically related and unrelated items, followed by short delay free and cued recall, long delay free and cued recall, and recognition test conditions [11]. The 12-word PVLT offers a number of key advantages over existing episodic memory assessments. First, PVLT items were derived from prototypical exemplars based upon a sample of cognitively normal community-dwelling older adult volunteers [9]. See Price et al. [9] (2009) and Bezdicek et al. [11] (2014) for more detailed descriptions of prototypical exemplar derivation. Briefly, community dwelling, non-patient research volunteers were given 2 minutes to write down as many objects (exemplars) as possible from different categories (e.g., fruit, office supplies, cleaning supplies). Exemplars (words) with moderate familiarity or prototypicality (words generated by $\sim 50 \%$ of the volunteers) were selected as the PVLT items. This selection process minimizes the chance that PVLT performance is influenced by item familiarity. Second, the PVLT comes with multiple versions, including three 9 -word versions that facilitate repeated assessment and 
minimize floor effects in more impaired patients and a 12-word list that was designed for detection of early stages of cognitive decline. Third, the PVLT offers a robust learning paradigm with five immediate free recall learning trials, a distractor trial, and cued recall trials that allows for the assessment of proactive and retroactive interference. Fourth, the recognition paradigm balances items from the original list (List A) and the distractor list (List B) with semantic and unrelated foils, allowing for generation of a more comprehensive set of error and recognition metrics. Finally, the PVLT is free and publicly available, has an alternate test form, and is a cost-effective tool for inclusion in both clinical and research settings.

However, critical validity data for the 12-word PVLT is lacking in older adult populations, thus limiting its utilization. Foremost, establishing a link between the 12-word PVLT and markers of unhealthy brain aging is essential for demonstrating this test's utility for identifying early pathology in older adults. Existing work suggests that retrieval deficits on the 9-word PVLT are associated with greater amounts of white matter damage, as assessed by MRI $[12,13]$. However, whether the tool correlates with pathology in brain regions necessary for learning and memory (e.g., medial temporal lobe) or is affected by pathological processes common in aging adults (e.g., amyloidosis, neurodegeneration) is not yet known. Additionally, normative data for the 12-word PVLT are limited, with the only published data being derived from a Czech population of older adults [11].

This study has several objectives. First, using a community sample of older adults aged 60-92 years and free of clinical dementia and stroke [14], we sought to identify biomarker correlates of performance patterns on the 12-word PVLT. We hypothesized that PVLT indices would relate to biomarker evidence of amyloid pathology measured by CSF A $\beta_{42}$ [4], neurodegeneration measured by brain MRI [6] and CSF total tau [4, 6, 15], WMH on brain MRI [16], and axonal injury assessed by CSF neurofilament light (NFL) [17]. Second, in a subset of participants with normal cognition (NC), we provide regression-based normative data accounting for demographic variables that often confound cognitive performance, including age, sex, and education [18]. The current study provides data supporting the utilization of the 12-word PVLT for assessing verbal episodic memory in older adults.

\section{Methods}

\section{Participants}

Participant data were drawn from the Vanderbilt Memory \& Aging Project, a longitudinal observational study investigating vascular health and brain aging, enriched for mild cognitive impairment (MCI) [14]. Participants were recruited from the community through postal mailings, radio advertisements, newsletters, research distribution emails, community events, websites, and word of mouth. Inclusion required participants be aged 60 years or older, native English speakers, have adequate auditory and visual acuity for testing purposes, and have a reliable study partner. At eligibility, participants underwent medical history and record review, clinical interview, including functional questionnaire [19] and Clinical Dementia Rating (CDR) [20] with the informant, and neuropsychological assessment. Participants were excluded for a cognitive diagnosis other than the following:

1 NC or cognitively unimpaired was defined as (a) CDR $=0$ (no dementia), (b) no activities of daily living deficits attributable to cognitive impairment, and (c) no objective neuropsychological impairment defined as standard scores falling 1.5 standard deviations within the age-adjusted normative mean.

2 Early MCI was defined as (a) CDR $=0.5$ (reflecting mild severity of impairment), (b) no activities of daily living deficits attributable to cognitive issues, and (c) no objective 


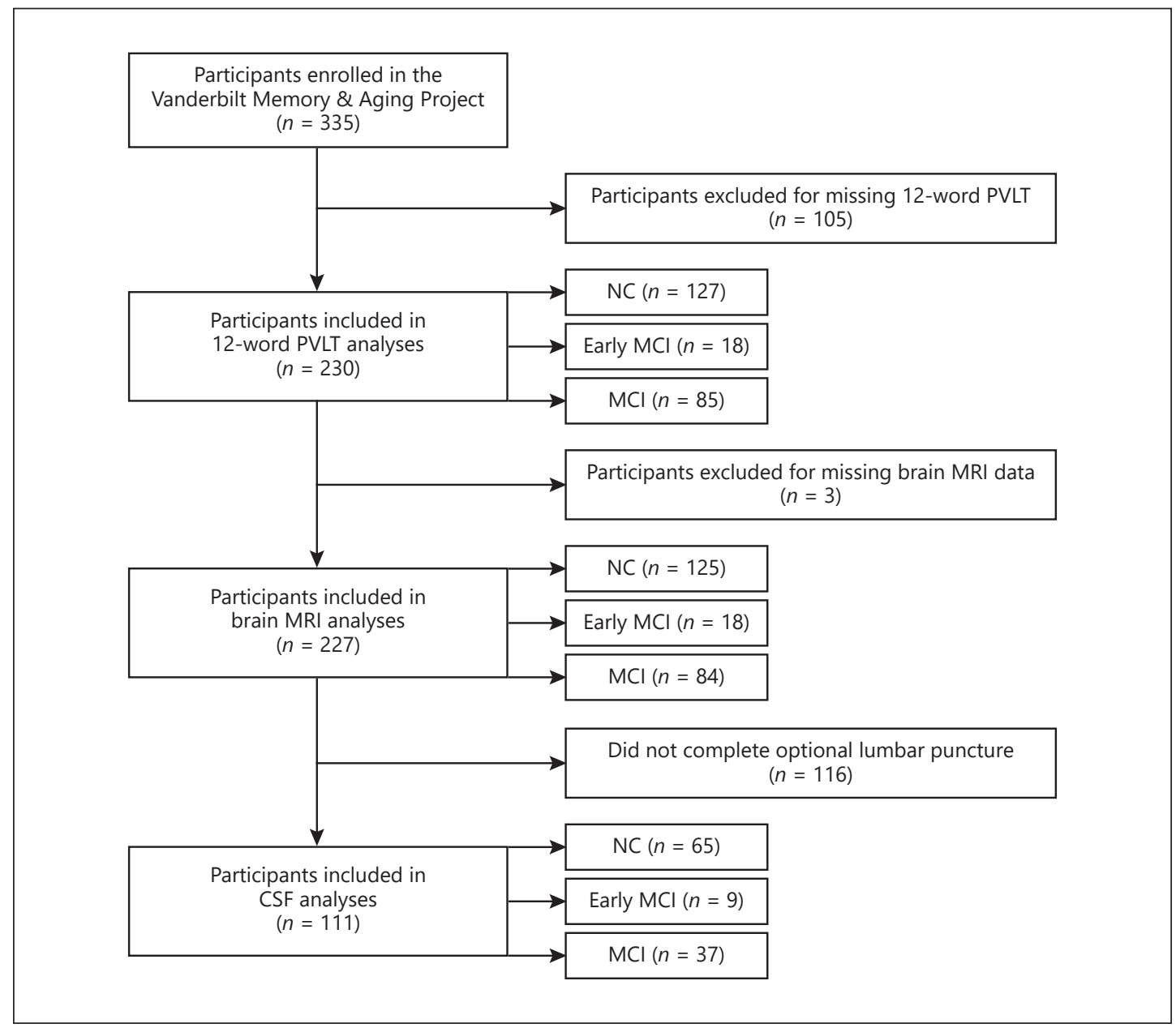

Fig. 1. Participant inclusion/exclusion criteria. Missing data categories are mutually exclusive. PVLT, 12word Philadelphia Verbal Learning Test; CSF, cerebrospinal fluid; NC, normal cognition; MCI, mild cognitive impairment.

neuropsychological impairment defined as standard scores falling 1.5 standard deviations within the age-adjusted normative mean [21].

3 MCI was defined as (a) CDR $=0$ or 0.5 (reflecting mild severity of impairment), (b) relatively spared activities of daily living, (c) objective neuropsychological impairment within at least one cognitive domain (i.e., performances falling greater than 1.5 standard deviations outside the age-adjusted normative mean or pre-morbid level of functioning), (d) concern of a cognitive change by the participant, informant, or clinician, and (e) absence of a dementing syndrome [3].

Additional exclusions included MRI contraindication, a history of neurological disease (e.g., dementia, multiple sclerosis, stroke), heart failure, major psychiatric illness, head injury with loss of consciousness $>5$ minutes, or a systemic or terminal illness affecting follow-up examination participation. At enrollment, participants completed a comprehensive evaluation, including clinical interview, neuropsychological assessment, and multi-modal brain MRI. A subset completed a lumbar puncture. For the purposes of this study, participants were excluded for missing 12-word PVLT data $(n=105)$, resulting in 230 participants aged 60-92 years (see Fig. 1). 


\section{Neuropsychological Assessment}

Participants completed a neuropsychological protocol that has been described previously [14]. Briefly, measures were carefully selected to preclude floor or ceiling effects and were not used to screen or select participants into the study. As part of this protocol, individuals completed a screener of depressive symptoms, the Geriatric Depression Scale [22]. Of note, the 12-word PVLT was administered as part of a separate neuropsychological protocol and PVLT scores were not used to determine diagnostic status. PVLT methods are described in the Introduction above and indices examined in the current study include List A Total Learning (across 5 learning trials), Short Delay Free Recall, Short Delay Cued Recall, Long Delay Free Recall, Long Delay Cued Recall, and Long Delay Recognition Total Discrimination. See Table 1 for details.

\section{Brain MRI}

Participants were scanned at the Vanderbilt University Institute of Imaging Science on a 3T Philips Achieva system (Best, The Netherlands) with 8-channel SENSE reception. Parameters and post-processing steps have been detailed elsewhere [14]. Briefly, T1-weighted MPRAGE images (isotropic spatial resolution $=1 \mathrm{~mm}^{3}$ ) were used to calculate regions of interest and intracranial volume using multi-atlas segmentation [23], and T2-weighted fluid-attenuated inversion recovery (FLAIR) images $\left(0.45 \times 0.45 \times 4 \mathrm{~mm}^{3}\right)$ were acquired for quantification of WMH and post-processed using the Lesion Segmentation Tool toolbox for SPM8 [24].

\section{CSF Acquisition}

A subset of individuals $(n=111)$ completed an optional morning fasting lumbar puncture. CSF was collected with polypropylene syringes using a Sprotte 25-gauge spinal needle in an intervertebral lumbar space. Samples were immediately mixed and centrifuged, and supernatants were aliquoted in $0.5-\mathrm{mL}$ polypropylene tubes and stored at $-80^{\circ} \mathrm{C}$. Samples were analyzed in batch using commercially available enzyme-linked immunosorbent assays (ELISA; Fujirebio, Ghent, Belgium) to determine levels of $A \beta_{42}$ (INNOTEST $^{\circledR} \beta-$ AMYLOID $\left._{(1-42)}\right)$ and total tau (INNOTEST ${ }^{\circledR}$ hTAU). NFL was measured using a commercially available ELISA (Uman Diagnostics, Umeå, Sweden). Processing was completed by board-certified laboratory technicians who were blinded to the clinical information [25]. Intra-assay CVs were $<10 \%$.

\section{Statistical Analysis}

Descriptive statistics were calculated for age, sex, self-reported race/ethnicity, education, mood - assessed with the Geriatric Depression Scale (GDS) total score [22], neuropsychological performances, CSF levels, brain MRI variables, and apolipoprotein E $\varepsilon 4$ (APOE- $\varepsilon 4)$ carrier status (positive: $\varepsilon 2 / \varepsilon 4, \varepsilon 3 / \varepsilon 4, \varepsilon 4 / \varepsilon 4$; negative: $\varepsilon 2 / \varepsilon 2, \varepsilon 2 / \varepsilon 3, \varepsilon 3 / \varepsilon 3$ ). To assess construct validity, linear regressions related PVLT indices (List A Total Learning, Short Delay Free Recall, Short Delay Cued Recall, Long Delay Free Recall, Long Delay Cued Recall, and Long Delay Recognition Total Discrimination) to brain MRI and CSF markers of brain health, including amyloidosis ( $\mathrm{CSF} A \beta_{42}$ ), neurodegeneration (hippocampal volumes, entorhinal cortex volumes, inferior lateral ventricle volumes, CSF total tau), log-transformed white matter macrostructure changes (WMH), and axonal injury (CSF NFL). Models were adjusted for age, sex, race/ethnicity, education, $A P O E$ - $\varepsilon 4$ carrier status, cognitive diagnosis, and intracranial volume (for brain MRI models). Significance was set a priori at $p<0.05$. Correction for multiple comparisons was performed using the false discovery rate (FDR) procedure and are presented alongside the uncorrected $p$ values. Exploratory models were repeated restricting the sample to NC only. 
Table 1.12-Word Philadelphia Verbal Learning Test: protocol and variable derivation

\begin{tabular}{|c|c|c|}
\hline Variable & Description & Range \\
\hline $\begin{array}{l}\text { PVLT List A Total Learning } \\
\text { (Trials } 1-5 \text { ) }\end{array}$ & Assesses learning for a set of 12 words across 5 learning trials & $0-60$ \\
\hline Trials 1-5 Primacy & $\begin{array}{l}\text { Number of words recalled from the beginning of the list (items 1-4; } \\
\text { primacy effect) }\end{array}$ & $0-20$ \\
\hline Trials 1-5 Middle & Number of words recalled from the middle of the list (items 5-8) & $0-20$ \\
\hline Trials 1-5 Recency & $\begin{array}{l}\text { Number of words recalled from the end of the list (items 9-12; recency } \\
\text { effect) }\end{array}$ & $0-20$ \\
\hline PVLT Distractor Trial (Trial B) & Assesses interference of learning a similar, novel list of 12 words & $0-12$ \\
\hline PVLT Short Delay Free Recall & $\begin{array}{l}\text { Assesses immediate free recall for a set of } 12 \text { words following } 5 \text { learning } \\
\text { trials and presentation of a } 12 \text {-item distractor trial (without re-exposure } \\
\text { to the original } 12 \text { test items) }\end{array}$ & $0-12$ \\
\hline PVLT Short Delay Cued Recall & Assesses immediate cued (grouping) recall for a set of 12 words & $0-12$ \\
\hline PVLT Long Delay Free Recall & $\begin{array}{l}\text { Assesses delayed recall for a set of } 12 \text { words following a } 20 \text {-minute filled } \\
\text { delay }\end{array}$ & $0-12$ \\
\hline PVLT Long Delay Cued Recall & $\begin{array}{l}\text { Assesses delayed cued (grouping) recall for a set of } 12 \text { words after a } \\
20 \text {-minute filled delay }\end{array}$ & $0-12$ \\
\hline $\begin{array}{l}\text { PVLT Long Delay Recognition } \\
\text { Total Correct }\end{array}$ & $\begin{array}{l}\text { Assesses recognition of the } 12 \text { words in a } 48 \text {-word randomized set after a } \\
20 \text {-minute filled delay }\end{array}$ & $0-12$ \\
\hline $\begin{array}{l}\text { PVLT Long Delay Recognition } \\
\text { Total False Alarms }\end{array}$ & $\begin{array}{l}\text { The number of total false positives (Distractor }+ \text { Semantic }+ \text { Unrelated) } \\
\text { recognized from a } 48 \text {-word randomized set after a } 20 \text {-minute filled delay }\end{array}$ & $0-36$ \\
\hline $\begin{array}{l}\text { PVLT Long Delay Recognition } \\
\text { Discriminability }\end{array}$ & $\begin{array}{l}\text { Assesses ability to recognize the list of } 12 \text { words from related and } \\
\text { nonrelated nontarget words after a } 20 \text {-minute filled delay ((Long Delay } \\
\text { Recognition Total Correct }+0.5) / 13)-((\text { Long Delay Recognition Total } \\
\text { False Alarms }+0.5) / 37)\end{array}$ & $0-1$ \\
\hline PVLT Repetitions & $\begin{array}{l}\text { The total number of word repetitions given across all trials (Trials 1-5, } \\
\text { Distractor, Short Delay Free/Cued, and Long Delay Free/Cued) }\end{array}$ & $\mathrm{n} / \mathrm{a}$ \\
\hline PVLT Intrusions & $\begin{array}{l}\text { The total number of incorrect words given across all trials (Trials 1-5, } \\
\text { Distractor, Short Delay Free/Cued, and Long Delay Free/Cued) }\end{array}$ & $\mathrm{n} / \mathrm{a}$ \\
\hline
\end{tabular}

Regression-based normative data were calculated from the NC and White/non-Hispanic subgroup using multiple regression analyses adjusting for age, sex, and education (common demographic confounders of episodic memory performance). Individuals who self-declared as non-White or Hispanic were excluded from the normative analyses due to the small sample size $(n=16)$ and resulting concerns about non-generalizability of the normative calculations among this small cohort. The raw scores on the PVLT indices were used as outcomes, including List A Total Learning, Distractor Trial, Short Delay Free Recall, Short Delay Cued Recall, Long Delay Free Recall, Long Delay Cued Recall, Long Delay Recognition Total Discrimination, and Total Intrusions (see Table 1). Sex was coded as male $=0$, female $=1$. Age and education in years were treated as continuous variables. Multi-collinearity was assessed by calculating variance inflation factors (VIF) and goodness of fit was assessed by visual inspection of residual plots for a functional form of the adjusting variables. Intercepts, $\beta$-coefficients, and 

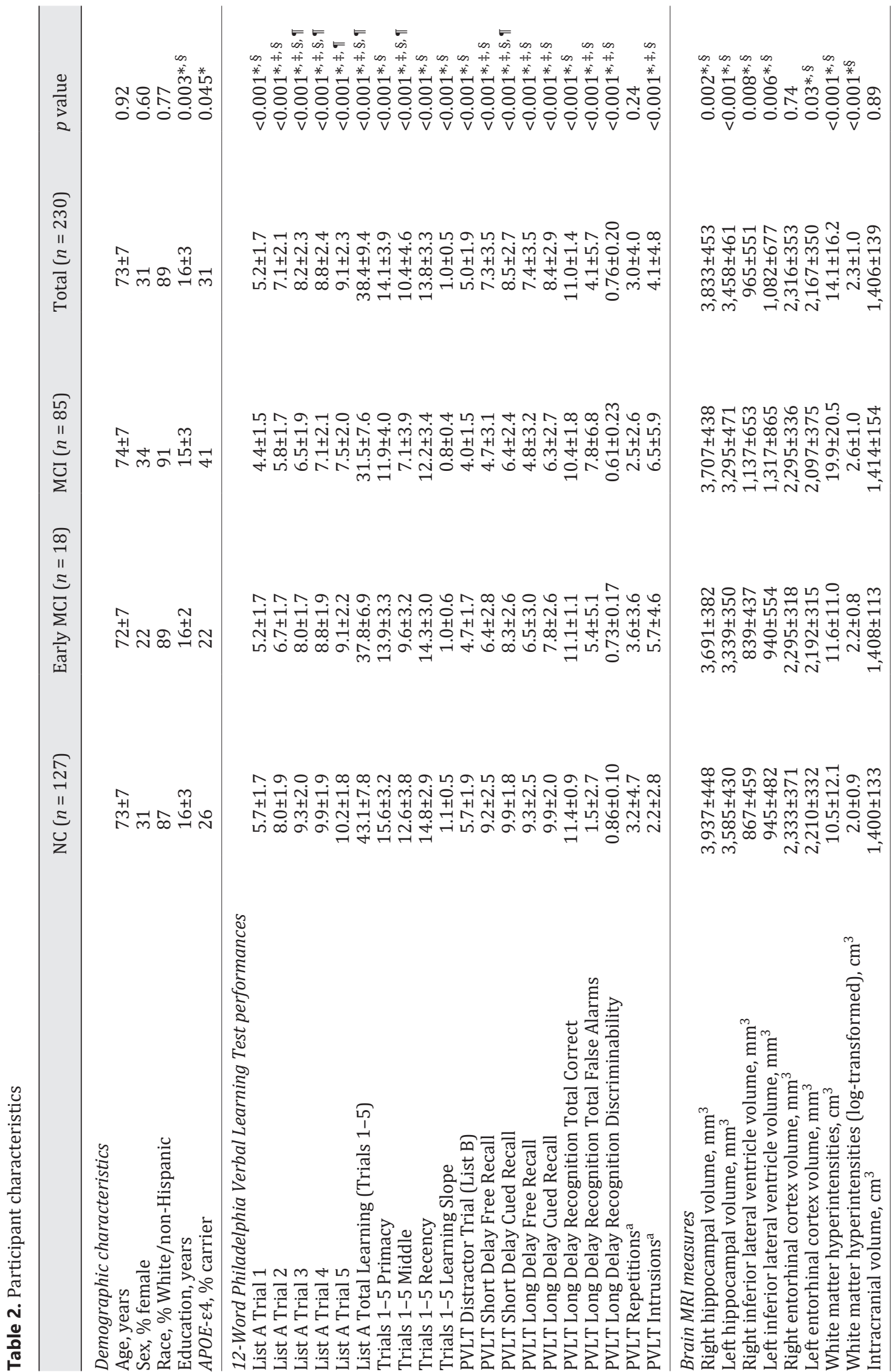

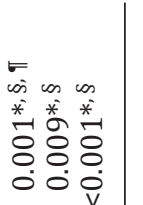

ह่

空

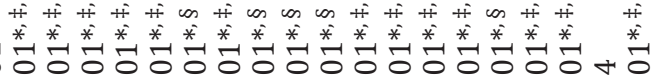

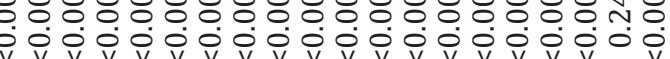

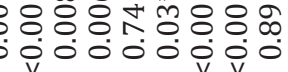

बे

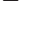


the root-mean-squared error for each model were calculated for derivation of a predicted PVLT performance value using the following equation (Equation 1):

Predicted score $=\beta_{0}$ (intercept $)+\beta_{\text {age }} \times$ age (years $)+\beta_{\text {sex }} \times \operatorname{sex}(0=$ male, $1=$ female $)+$ $\beta_{\text {education }} \times$ education (years).

To calculate a z-score normative value, the predicted score calculated in Equation 1 was used within the following equation (Equation 2) [26]:

z-score $=($ observed score - predicted score $) /$ root-mean-squared error .

All analyses were conducted using R 3.4.3 (www.r-project.org).

\section{Results}

\section{Participant Characteristics}

A total of 230 participants were included ranging in age from 60 to 92 years $(73 \pm 7$ years), including $31 \%$ female. A majority of participants self-declared as White/non-Hispanic (89\%). The entire sample had an education range from 7 to 20 years (16 \pm 3 years). Global cognition as assessed by the MoCA ranged from 14 to 30 (25 \pm 3 ). See Table 2 for participant characteristics and 12-word PVLT performances presented by the diagnostic groups. A subsample ( $n=111$ ) completed lumbar puncture ( $73 \pm 7$ years), including $24 \%$ female, $95 \%$ self-declared White/non-Hispanic, and education of $16 \pm 3$ years. The group completing a lumbar puncture had more males and White/non-Hispanic participants compared to the group who did not complete a lumbar puncture (see online suppl. Table 1 for full characteristics; for all online suppl. material, see www.karger.com/doi/10.1159/000494209).

\section{2-Word PVLT and Markers of Brain Health (Entire Cohort)}

Amyloidosis

Worse performance on only PVLT List A Total Learning was associated with lower CSF $\mathrm{A} \beta_{42}$ levels $(p=0.02$; FDR corrected $=0.04)$. See Table 3 for details.

Neurodegeneration

Worse performance on all PVLT indices was associated with higher CSF total tau levels $(p<0.04$; FDR corrected $=0.01-0.06)$. Worse performance on all PVLT indices was associated with smaller left hippocampal $(p<0.004$; FDR corrected $=0.01-0.02)$, smaller left entorhinal cortex (all $p<0.01$; FDR corrected $=0.01-0.02$ ), and greater (reflecting more neurodegeneration) left inferior lateral ventricle $(p<0.03$; FDR corrected $=0.01-0.049)$ volumes. Worse performance on most PVLT indices was also associated with smaller right hippocampal volume $(p<0.03$; FDR corrected $=0.04-0.049)$ with the exception of PVLT Long Delay Free Recall $(p=0.06)$ and Long Delay Recognition Total Discrimination $(p=0.09)$. Worse performance on all PVLT indices was associated with smaller right entorhinal cortex volume $(p<$ 0.04; FDR corrected $=0.01-0.06$ ). Worse performance on most PVLT indices was associated with larger right inferior lateral ventricle volume $(p<0.05$; FDR corrected $=0.01-0.049)$ with the exception of Short Delay and Long Delay Cued Recalls $(p>0.06)$. See Table 3 for details.

Axonal Integrity and White Matter Disease

Worse performance on PVLT Long Delay Cued Recall was only associated with higher CSF NFL levels $(p=0.03$; FDR corrected $=0.049)$. Worse performance on PVLT Short Delay Free 
Gifford et al.: PVLT Performance in Older Adults

Table 3. 12-Word Philadelphia Verbal Learning Test in relation to brain and CSF biomarkers: entire cohort

\begin{tabular}{|c|c|c|c|c|c|c|}
\hline & \multicolumn{2}{|c|}{$\begin{array}{l}\text { PVLT List A Total } \\
\text { Learning }\end{array}$} & \multicolumn{2}{|c|}{$\begin{array}{l}\text { PVLT Short Delay } \\
\text { Free Recall }\end{array}$} & \multicolumn{2}{|c|}{$\begin{array}{l}\text { PVLT Short Delay } \\
\text { Cued Recall }\end{array}$} \\
\hline & $\beta$ & $p$ value & $\beta$ & $p$ value & $\beta$ & $p$ value \\
\hline $\mathrm{CSF} A \beta_{42}$ & 5.96 & $0.02^{*}$ & 2.12 & 0.78 & 13.82 & 0.15 \\
\hline CSF T-tau & -6.53 & $0.008^{*}$ & -17.41 & $0.01^{*}$ & -20.22 & $0.02 *$ \\
\hline CSF NFL & -11.27 & 0.11 & -31.04 & 0.12 & -32.01 & 0.21 \\
\hline Right hippocampus volume & 8.36 & $0.02^{*}$ & 20.73 & $0.03^{*}$ & 25.98 & $0.03^{*}$ \\
\hline Left hippocampus volume & 10.64 & $0.004^{*}$ & 35.48 & $<0.001^{*}$ & 48.25 & $<0.001^{*}$ \\
\hline Right entorhinal cortex volume & 6.43 & $0.03^{*}$ & 17.23 & $0.04^{*}$ & 33.73 & $0.001^{*}$ \\
\hline Left entorhinal cortex volume & 8.25 & $0.005^{*}$ & 20.53 & $0.01^{*}$ & 40.21 & $<0.001^{*}$ \\
\hline Right inferior lateral ventricle volume & -10.01 & $0.03^{*}$ & -25.93 & $0.04^{*}$ & -30.45 & 0.06 \\
\hline Left inferior lateral ventricle volume & -16.71 & $0.004^{*}$ & -37.60 & $0.02^{*}$ & -60.63 & $0.002^{*}$ \\
\hline \multirow[t]{3}{*}{ WMH volume } & -0.01 & 0.12 & -0.05 & $0.048^{*}$ & -0.05 & 0.10 \\
\hline & \multicolumn{2}{|c|}{$\begin{array}{l}\text { PVLT Long Delay } \\
\text { Free Recall }\end{array}$} & \multicolumn{2}{|c|}{$\begin{array}{l}\text { PVLT Long Delay } \\
\text { Cued Recall }\end{array}$} & \multicolumn{2}{|c|}{$\begin{array}{l}\text { PVLT } \\
\text { Discrimination }\end{array}$} \\
\hline & $\beta$ & $p$ value & $\beta$ & $p$ value & $\beta$ & $p$ value \\
\hline $\mathrm{CSF} A \beta_{42}$ & 8.65 & 0.21 & 7.20 & 0.42 & 257.34 & 0.07 \\
\hline CSF T-tau & -13.70 & $0.03^{*}$ & -17.39 & $0.04^{*}$ & -406.58 & $0.002 *$ \\
\hline CSF NFL & -34.27 & 0.06 & -51.94 & $0.03^{*}$ & -174.11 & 0.65 \\
\hline Right hippocampus volume & 16.99 & 0.06 & 24.22 & $0.03^{*}$ & 261.65 & 0.09 \\
\hline Left hippocampus volume & 39.30 & $<0.001^{*}$ & 45.17 & $<0.001^{*}$ & 589.50 & $<0.001^{*}$ \\
\hline Right entorhinal cortex volume & 23.51 & $0.002^{*}$ & 29.54 & $0.002^{*}$ & 440.45 & $<0.001^{*}$ \\
\hline Left entorhinal cortex volume & 24.81 & $0.001 *$ & 36.60 & $<0.001^{*}$ & 413.95 & $0.002 *$ \\
\hline Right inferior lateral ventricle volume & -23.77 & $0.049 *$ & -24.36 & 0.10 & -428.12 & $0.04^{*}$ \\
\hline Left inferior lateral ventricle volume & -37.93 & $0.01^{*}$ & -40.29 & $0.03^{*}$ & -828.77 & $0.001^{*}$ \\
\hline WMH volume & -0.05 & $0.01^{*}$ & -0.05 & $0.046^{*}$ & -0.41 & 0.28 \\
\hline
\end{tabular}

PVLT, 12-word Philadelphia Verbal Learning Test; CSF, cerebrospinal fluid; A $\beta$, amyloid-beta; T-tau, total tau; NFL, neurofilament light; WMH, white matter hyperintensity (log-transformed). * Significance threshold at $p<0.05$.

Recall, Long Delay Free Recall, and Long Delay Cued Recall was associated with greater WMH $(p<0.05$; FDR corrected $=0.02-0.07)$. See Table 3 for details.

\section{2-Word PVLT and Markers of Brain Health: Exploratory Analyses in NC Only}

Secondary exploratory analyses repeating models limited to NC participants revealed no associations between any PVLT variable and CSF A $\beta_{42}$ or total tau $(p>0.19)$. See Table 4 for details.

Worse performance on PVLT Long Delay Free Recall $(p=0.02)$ was associated with smaller left hippocampal volume. PVLT indices were not related to any other brain region of interest (all $p>0.09$ ). See Table 4 for details.

Worse performance on all PVLT indices (all $p<0.03$ ) was associated with higher CSF NFL. Additionally, worse performance on PVLT Short Delay Cued Recall, PVLT Long Delay Free Recall, and PVLT Long Delay Recognition Total Discrimination $(p<0.05)$ was associated with greater WMH burden. See Table 4 for details.

\section{2-Word PVLT Regression-Based Normative Data}

Review of VIF revealed no multicollinearity between demographic variables (all VIF < 1.4). The residuals plotted against predicted values did not reveal any systematic patterns, 
Gifford et al.: PVLT Performance in Older Adults

Table 4. 12-Word Philadelphia Verbal Learning Test in relation to brain and CSF biomarkers: cognitively normal cohort

\begin{tabular}{|c|c|c|c|c|c|c|}
\hline & \multicolumn{2}{|c|}{$\begin{array}{l}\text { PVLT List A Total } \\
\text { Learning }\end{array}$} & \multicolumn{2}{|c|}{$\begin{array}{l}\text { PVLT Short Delay } \\
\text { Free Recall }\end{array}$} & \multicolumn{2}{|c|}{$\begin{array}{l}\text { PVLT Short Delay } \\
\text { Cued Recall }\end{array}$} \\
\hline & $\beta$ & $p$ value & $\beta$ & $p$ value & $\beta$ & $p$ value \\
\hline $\operatorname{CSF} A \beta_{42}$ & 4.55 & 0.23 & 7.00 & 0.56 & 21.48 & 0.19 \\
\hline CSF T-tau & -2.35 & 0.44 & -6.87 & 0.47 & -3.05 & 0.82 \\
\hline CSF NFL & -21.58 & $0.003^{*}$ & -64.18 & $0.004^{*}$ & -76.60 & $0.02^{*}$ \\
\hline Right hippocampus volume & 3.13 & 0.50 & 19.27 & 0.17 & 3.02 & 0.87 \\
\hline Left hippocampus volume & 4.16 & 0.34 & 22.27 & 0.09 & 12.51 & 0.48 \\
\hline Right entorhinal cortex volume & -0.06 & 0.99 & 8.84 & 0.48 & 17.23 & 0.31 \\
\hline Left entorhinal cortex volume & -0.67 & 0.85 & 7.11 & 0.49 & 13.00 & 0.36 \\
\hline Right inferior lateral ventricle volume & 2.35 & 0.68 & 0.99 & 0.95 & -2.97 & 0.90 \\
\hline Left inferior lateral ventricle volume & 0.54 & 0.93 & 5.63 & 0.76 & 8.85 & 0.72 \\
\hline \multirow[t]{3}{*}{ WMH volume } & -0.01 & 0.32 & -0.05 & 0.15 & -0.10 & $0.03^{*}$ \\
\hline & \multicolumn{2}{|c|}{$\begin{array}{l}\text { PVLT Long Delay } \\
\text { Free Recall }\end{array}$} & \multicolumn{2}{|c|}{$\begin{array}{l}\text { PVLT Long Delay } \\
\text { Cued Recall }\end{array}$} & \multicolumn{2}{|c|}{$\begin{array}{l}\text { PVLT } \\
\text { Discrimination }\end{array}$} \\
\hline & $\beta$ & $p$ value & $\beta$ & $p$ value & $\beta$ & $p$ value \\
\hline $\mathrm{CSF} A \beta_{42}$ & 5.88 & 0.62 & 9.88 & 0.53 & -53.49 & 0.86 \\
\hline CSF T-tau & 5.18 & 0.58 & -2.07 & 0.87 & 181.36 & 0.44 \\
\hline CSF NFL & -58.21 & $0.01^{*}$ & -68.76 & $0.02 *$ & $-1,286.67$ & $0.03^{*}$ \\
\hline Right hippocampus volume & 15.99 & 0.25 & 5.61 & 0.75 & 375.52 & 0.28 \\
\hline Left hippocampus volume & 29.61 & $0.02 *$ & 17.14 & 0.29 & 587.23 & 0.07 \\
\hline Right entorhinal cortex volume & 18.19 & 0.14 & 24.81 & 0.11 & 388.41 & 0.21 \\
\hline Left entorhinal cortex volume & 12.57 & 0.22 & 14.45 & 0.26 & 254.91 & 0.31 \\
\hline Right inferior lateral ventricle volume & -3.89 & 0.82 & 1.20 & 0.96 & -129.82 & 0.76 \\
\hline Left inferior lateral ventricle volume & -0.46 & 0.98 & 5.76 & 0.80 & 60.24 & 0.89 \\
\hline WMH volume & -0.07 & $0.03^{*}$ & -0.07 & 0.09 & -1.70 & $0.045^{*}$ \\
\hline
\end{tabular}

PVLT, 12-word Philadelphia Verbal Learning Test; CSF, cerebrospinal fluid; A $\beta$, amyloid-beta; T-tau, total tau; NFL, neurofilament light; WMH, white matter hyperintensity (log-transformed). * Significance threshold at $p<0.05$.

suggesting an appropriate functional form of demographic variables and goodness of fit. Means, intercepts, and regression coefficients are presented in Table 5 for transforming raw scores into demographically adjusted z-scores using Equations 1 and 2 in Statistical Analysis.

For illustrative purposes, normative data for PVLT Total Learning were calculated for a 75-year old woman with 16 years of education. Using Equation 1, the predicted score was calculated as follows:

56.71 (PVLT Total Learning intercept) $+-0.40 \times 75$ (PVLT Total Learning $\beta_{\text {age }} \times$ actual age) $+7.71 \times 1$ (PVLT Total Learning $\beta_{\text {sex }} \times 1=$ female $)+0.85 \times 16$ (PVLT Total Learning $\beta_{\text {education }} \times$ number of years of education completed) $=48.02$.

To calculate a normative value with an obtained score on PVLT Total Learning of 36, Equation 2 is used:

(36 [observed score] - 48.02 [predicted score])/6.86 [root-mean-squared error for PVLT Total Learning] for a resultant z-score of -1.75 .

In this illustration, a total learning score of 36 for a 75 -year old woman with a college education would be impaired at $\mathrm{z}=-1.75$. 
Gifford et al.: PVLT Performance in Older Adults

Table 5. Mean and regression coefficients for normative data calculation

\begin{tabular}{lcccccc}
\hline & Mean \pm SD & Intercept & $\beta$ (age) & $\beta$ (sex) & $\beta$ (education) & RMSE \\
\hline PVLT List A Total Learning & $43.1 \pm 7.8$ & $56.71^{* * *}$ & $-0.40^{* * *}$ & $7.71^{* * *}$ & $0.85^{* *}$ & 6.86 \\
PVLT Distractor Trial & $5.7 \pm 1.9$ & $9.99^{* * *}$ & $-0.08^{* *}$ & $1.44^{* * *}$ & 0.07 & 1.73 \\
PVLT Short Delay Free Recall & $9.2 \pm 2.5$ & $13.37^{* * *}$ & $-0.12^{* * *}$ & $1.92^{* * *}$ & $0.27^{* *}$ & 2.26 \\
PVLT Short Delay Cued Recall & $9.9 \pm 1.8$ & $10.36^{* * *}$ & $-0.06^{*}$ & $1.77^{* * *}$ & $0.20^{* *}$ & 1.67 \\
PVLT Long Delay Free Recall & $9.3 \pm 2.5$ & $13.44^{* * *}$ & $-0.12^{* * *}$ & $2.28^{* * *}$ & $0.23^{*}$ & 2.22 \\
PVLT Long Delay Cued Recall & $9.9 \pm 2.0$ & $12.01^{* * *}$ & $-0.08^{* *}$ & $1.82^{* * *}$ & $0.18^{*}$ & 1.75 \\
PVLT Total Discrimination & $0.86 \pm 0.10$ & $1.09^{* * *}$ & $-0.006^{* * *}$ & $0.09^{* * *}$ & $0.01^{* *}$ & 0.09 \\
PVLT Total Intrusions & $2.2 \pm 2.8$ & -0.59 & 0.06 & -0.58 & -0.08 & 2.82 \\
\hline
\end{tabular}

PVLT, Philadelphia Verbal Learning Test; RMSE, root-mean-squared error. ${ }^{*} p<0.05,{ }^{* *} p<0.01,{ }^{* * *} p<0.001$.

\section{Discussion}

The current study assessed the validity of the 12-word PVLT, a verbal list-learning episodic memory test, and offers robust normative data for increased clinical utility among older adults. When our sample was analyzed as a whole, worse performance on nearly all of the 12-word PVLT indices was related to increased CSF total tau and smaller left medial temporal lobe brain volumes, whereas fewer associations were noted with white matter macrostructure or amyloidosis. However, when analyzing only NC older adult performances, worse PVLT performance was broadly related to increased axonal injury and white matter macrostructure damage.

These results are among the first to highlight that the 12-word PVLT performances are associated with medial temporal lobe structures critical for intact learning and memory [27, 28]. Specifically, the PVLT was broadly related to atrophy of the medial temporal lobe, including the hippocampus and entorhinal cortex, on brain MRI. This regional pattern of atrophy has been linked to episodic memory decline [6], presumably because they are the first regions to be affected by AD pathology [29-31]. PVLT performance was also related to CSF total tau, a biomarker of neurodegeneration. Tau pathology, including neurofibrillary tangles associated with $\mathrm{AD}$, begins in the entorhinal cortex and adjacent limbic structures with evolution into the cortex [32], resulting in axonal loss and neurodegeneration. Tissue volume loss is closely related to CSF total tau [33] and correlated with cognition and disease severity [34], which aligns with the current results suggesting performance on the 12-word PVLT correlates with underlying neurodegeneration. Results remain largely unchanged after correction for multiple comparisons. Overall, the 12-word PVLT appears to have good construct validity for assessing structural integrity of the medial temporal lobe and may be an important tool for the assessment of neurodegenerative disorders.

A different pattern of associations emerged in exploratory analyses including only cognitively normal individuals. Specifically, lower performance on the 12-word PVLT was associated with increased white matter macrostructure damage (WMH) and poorer axonal health (CSF NFL), suggesting these white matter integrity markers are preferentially important to cognition in NC older adults. Prior literature has linked greater WMH to reduced verbal episodic memory performance in cognitively normal older adults [35], consistent with the current results. The NFL association in cognitively normal participants represents a novel finding but is in line with previous research linking NFL and WMH [17, 36, 37]. Collectively, these results might represent age-related changes given that WMH burden increases with age [38] and is associated with age-related decline in verbal episodic memory [39]. Furthermore, these episodic memory changes that occur prior to overt cognitive impairment appear to be 
preferentially related to white matter integrity [40] as compared to cortical thickness [41]. Similarly, CSF NFL is hypothesized to be indicative of damage to large-caliber myelinated axons and microstructural integrity within the cerebral white matter [42, 43] that may be highly relevant to cognition prior to clinical impairment. Plus, prior research has linked CSF NFL and WMH burden, including data from this cohort. Alternatively, these results may highlight a non-amyloid pathway to cognitive changes [44,45], such as cerebral small vessel disease [46, 47]. Regardless of etiology, these findings add to the existing literature and support the importance of white matter health in episodic memory in the absence of, or prior to, overt evidence of clinical or pathological AD. However, given the exploratory nature of the current analyses, these findings require replication.

The limited association between PVLT and CSF amyloid in the entire group and the null associations between PVLT and CSF amyloid in the NC group warrant brief discussion. Structural or CSF markers of neurodegeneration more strongly relate to cognition than amyloidosis [48-50]. Thus, the limited associations reported here between PVLT and CSF amyloid may be due to the temporal nature of AD pathology, with amyloid levels increasing years prior to the onset of neuronal loss and cognitive decline [51-53]. Similarly, the lack of associations between the 12-word PVLT and brain and CSF markers of neurodegeneration in the $\mathrm{NC}$ group may have been related to limited pathology in this group or insufficient power to detect differences due to the smaller sample size.

Advancing age was related to poorer performance on all PVLT indices, consistent with previous research linking increasing age with decline in verbal memory $[18,54]$. Sex and education were generally related to the PVLT indices, with overall results aligning with previous research suggesting better cognitive performance is related to more years of education and female sex [18]. Given these potential demographic confounds on task performance, the regression-based normative data provided here incorporate all of these demographic factors, allowing for more robust generation of normative data. Of note, race and ethnicity are known confounders of cognitive performance [18]; however, the current study was unable to thoroughly consider race or ethnicity in normative data calculations due to limited diversity in the sample $(n=16)$. We excluded these participants a priori from the regression-based normative data calculations to prevent the normative information published here from being incorrectly applied to diverse populations (potentially leading to incorrect interpretation of patient performances). This exclusion of participants (and lack of representation in the sample) limits the utilization of this tool, and future work should emphasize expanding the normative data to represent more racially diverse cohorts.

The current study has several strengths. First, this study is the first to link 12-word PVLT performances with various biomarkers of brain health. Similarly, we are among the first to examine the cognitive correlates of CSF NFL in cognitively normal older adults using a comprehensive episodic memory paradigm. Second, the current study provides older adult normative data for the 12-word PVLT for the first time, emphasizing regression-based methodology that incorporates multiple demographic confounds. Finally, all enrolled participants underwent an extensive phenotyping of cognitive status, including CDR interview, medical record and health history review, comprehensive neuropsychological protocol, and consensus decision for diagnostic status. Of note, PVLT performance was not part of the diagnostic determinations; it was administered in a separate testing session.

Despite these strengths, several limitations warrant consideration. First, aspects of the cohort limit generalizability of the results. For example, the sample is predominantly White with a mean college education level. The normative data provided are for White/non-Hispanic individuals due to the small sample of non-White/Hispanic individuals and concerns about non-generalizability of the normative values. These factors should be considered when using the presented normative data and it is essential that future research provide normative data 
for younger cohorts and more racially/ethnically diverse cohorts to increase generalization and utility of the 12-word PVLT. Second, the use of CSF biomarkers limits our ability to speak to regional associations of amyloid or tau deposition. Third, as this study is cross-sectional and without longitudinal information, we are unable to confirm that the cognitively normal participants do not have underlying neuropathology that ultimately leads to $\mathrm{MCI}$ or dementia conversion. Next, the PVLT items were selected from a single sample within one geographic location. It is possible that the typicality of these words differs between different countries; however, efforts are ongoing to address this limitation and construct PVLT versions in Mandarin, Czech, Spanish, and Farsi. Lastly, the cohort used to derive normative data has a smaller sample size in comparison to previous normative research with other learning and memory tests $[55,56]$. However, regression-based normative procedures require smaller samples sizes than traditional normative procedures [57] and allow for simultaneous consideration of multiple factors known to influence cognitive performance. The current study represents a first effort for assessing validity and providing normative data, but future research is needed to replicate these findings with a larger sample.

Overall, this study is among the first to enhance the clinical utility of the 12-word PVLT, an episodic memory tool that assesses learning, encoding, retrieval, recognition, and freedom from interference. Results include regression-based normative data based upon age, sex, and education to enhance the clinical utility of this promising verbal episodic memory test. Furthermore, results suggest the PVLT has good psychometric properties and relates to brain health biomarkers, including medial temporal lobe integrity. The 12-word PVLT also appears to be sensitive to very early cognitive changes, prior to overt cognitive impairment. Longitudinal research is needed to examine the prognostic utility of the 12-word PVLT and to determine if associations represent age-related processes, non-AD pathological processes common in aging (e.g., small vessel disease), or very early precursors to AD.

\section{Acknowledgments}

We thank the patients and their families, whose help and participation made this work possible. This work was supported by the Paul B. Beeson Career Development Award in Aging K23-AG030962 (A.L.J.); K24-AG046373 (A.L.J.); Alzheimer's Association IIRG-08-88733 (A.L.J);R01-AG034962(A.L.J.);R01-AG056534(A.L.J.);R01-NS100980 (A.L.J.);K12-HD043483 (K.A.G., T.J.H., S.P.B.); Alzheimer's Association NIRG-13-283276 (K.A.G.); K23-AG045966 (K.A.G.); Paul B. Beeson Career Development Award in Aging K23-AG048347 (S.P.B.); the Eisenstein Women's Heart Fund (S.B.P.); K01-AG049164 (T.J.H.); F32-AG058395 (K.E.O.); Vanderbilt Institute for Clinical and Translational Research UL1-TR000445; Vanderbilt's High-Performance Computer Cluster for Biomedical Research S10-OD023680; the Vanderbilt Memory and Alzheimer's Center; the Swedish Research Council; the Swedish Alzheimer's Association; the Knut and Alice Wallenberg Foundation, and Torsten Söderberg Foundation, Stockholm, Sweden.

\section{Statement of Ethics}

The Vanderbilt University Medical Center Institutional Review Board approved the protocol. Written informed consent was obtained from all participants prior to data collection. 
Gifford et al.: PVLT Performance in Older Adults

\section{Disclosure Statement}

All authors declare no conflicts of interest in relation to the current work. H.Z. has served at advisory boards for Eli Lilly and Roche Diagnostics, has received travel support from Teva, and is a co-founder of Brain Biomarker Solutions in Gothenburg AB, a GU Ventures-based platform company at the University of Gothenburg.

\section{References}

1 Albert MS, Moss MB, Tanzi R, Jones K. Preclinical prediction of AD using neuropsychological tests. J Int Neuropsychol Soc. 2001 Jul; 7(5):631-9.

2 Jedynak BM, Lang A, Liu B, Katz E, Zhang Y, Wyman BT, et al.; Alzheimer's Disease Neuroimaging Initiative. A computational neurodegenerative disease progression score: method and results with the Alzheimer's disease Neuroimaging Initiative cohort. Neuroimage. 2012 Nov;63(3):1478-86.

3 Albert MS, DeKosky ST, Dickson D, Dubois B, Feldman HH, Fox NC, et al. The diagnosis of mild cognitive impairment due to Alzheimer's disease: recommendations from the National Institute on Aging-Alzheimer's Association workgroups on diagnostic guidelines for Alzheimer's disease. Alzheimers Dement. 2011 May; $7(3): 270-9$.

4 Mandecka M, Budziszewska M, Barczak A, Pepłońska B, Chodakowska-Żebrowska M, Filipek-Gliszczyńska A, et al. Association between cerebrospinal fluid biomarkers for Alzheimer's disease, APOE genotypes and auditory verbal learning task in subjective cognitive decline, mild cognitive impairment, and Alzheimer's disease. J Alzheimers Dis. 2016 Jul;54(1):157-68.

5 Resnick SM, Sojkova J, Zhou Y, An Y, Ye W, Holt DP, et al. Longitudinal cognitive decline is associated with fibrillar amyloid-beta measured by [11C]PiB. Neurology. 2010 Mar;74(10):807-15.

6 Wolk DA, Dickerson BC; Alzheimer's Disease Neuroimaging Initiative. Fractionating verbal episodic memory in Alzheimer's disease. Neuroimage. 2011 Jan;54(2):1530-9.

7 Li D, Shen D, Tai H, Cui L. Neurofilaments in CSF as diagnostic biomarkers in motor neuron disease: a metaanalysis. Front Aging Neurosci. 2016 Nov; 8:290.

8 Delis DC, Kramer JH, Kaplan E, Ober BA. California Verbal Learning Test: Adult Version. San Antonio (TX): The Psychological Corporation; 1987.

9 Price CC, Garrett KD, Jefferson AL, Cosentino S, Tanner JJ, Penney DL, et al. Leukoaraiosis severity and listlearning in dementia. Clin Neuropsychol. 2009 Aug;23(6):944-61.

10 Garrett KD, Price CC, Lamar M, Giovannetti T, Delano-Wood L, Penny DL, et al. Assessing verbal and visual serial learning: The California Verbal Learning Test and the Philadelphia (Repeatable) Verbal Learning Test. In: Ashendorf L, Swenson R, Libon DJ, editors. The Boston Process Approach to Neuropsychological Assessment: A Practitioner's Guide. New York: Oxford University Press; 2013. p. 148-69.

11 Bezdicek O, Libon DJ, Stepankova H, Panenkova E, Lukavsky J, Garrett KD, et al. Development, validity, and normative data study for the 12-word Philadelphia Verbal Learning Test [czP(r)VLT-12] among older and very old Czech adults. Clin Neuropsychol. 2014;28(7):1162-81.

12 Price CC, Jefferson AL, Merino JG, Heilman KM, Libon DJ. Subcortical vascular dementia: integrating neuropsychological and neuroradiologic data. Neurology. 2005 Aug;65(3):376-82.

13 Libon DJ, Price CC, Giovannetti T, Swenson R, Bettcher BM, Heilman KM, et al. Linking MRI hyperintensities with patterns of neuropsychological impairment: evidence for a threshold effect. Stroke. 2008 Mar;39(3): 806-13.

14 Jefferson AL, Gifford KA, Acosta LM, Bell SP, Donahue MJ, Davis LT, et al. The Vanderbilt Memory \& Aging Project: study design and baseline cohort overview. J Alzheimers Dis. 2016 Mar;52(2):539-59.

15 Murphy EA, Holland D, Donohue M, McEvoy LK, Hagler DJ Jr, Dale AM, et al.; Alzheimer's Disease Neuroimaging Initiative. Six-month atrophy in MTL structures is associated with subsequent memory decline in elderly controls. Neuroimage. 2010 Dec;53(4):1310-7.

16 Lee S, Viqar F, Zimmerman ME, Narkhede A, Tosto G, Benzinger TL, et al.; Dominantly Inherited Alzheimer Network. White matter hyperintensities are a core feature of Alzheimer's disease: evidence from the dominantly inherited Alzheimer network. Ann Neurol. 2016 Jun;79(6):929-39.

17 Zetterberg H, Skillbäck T, Mattsson N, Trojanowski JQ, Portelius E, Shaw LM, et al.; Alzheimer's Disease Neuroimaging Initiative. Association of cerebrospinal fluid neurofilament light concentration with Alzheimer disease progression. JAMA Neurol. 2016 Jan;73(1):60-7.

18 Norman MA, Evans JD, Miller WS, Heaton RK. Demographically corrected norms for the California Verbal Learning Test. J Clin Exp Neuropsychol. 2000 Feb;22(1):80-94.

19 Pfeffer RI, Kurosaki TT, Harrah CH Jr, Chance JM, Filos S. Measurement of functional activities in older adults in the community. J Gerontol. 1982 May;37(3):323-9.

20 Morris JC. The Clinical Dementia Rating (CDR): current version and scoring rules. Neurology. 1993 Nov; 43(11):2412-4. 
21 Aisen PS, Petersen RC, Donohue MC, Gamst A, Raman R, Thomas RG, et al.; Alzheimer's Disease Neuroimaging Initiative. Clinical Core of the Alzheimer's Disease Neuroimaging Initiative: progress and plans. Alzheimers Dement. 2010 May;6(3):239-46.

22 Yesavage JA, Brink TL, Rose TL, Lum O, Huang V, Adey M, et al. Development and validation of a geriatric depression screening scale: a preliminary report. J Psychiatr Res. 1982-1983;17(1):37-49.

23 Asman AJ, Landman BA. Formulating spatially varying performance in the statistical fusion framework. IEEE Trans Med Imaging. 2012 Jun;31(6):1326-36.

24 Schmidt P, Gaser C, Arsic M, Buck D, Förschler A, Berthele A, et al. An automated tool for detection of FLAIRhyperintense white-matter lesions in Multiple Sclerosis. Neuroimage. 2012 Feb;59(4):3774-83.

25 Palmqvist S, Zetterberg H, Blennow K, Vestberg S, Andreasson U, Brooks DJ, et al. Accuracy of brain amyloid detection in clinical practice using cerebrospinal fluid $\beta$-amyloid 42: a cross-validation study against amyloid positron emission tomography. JAMA Neurol. 2014 Oct;71(10):1282-9.

26 Shirk SD, Mitchell MB, Shaughnessy LW, Sherman JC, Locascio JJ, Weintraub S, et al. A web-based normative calculator for the uniform data set (UDS) neuropsychological test battery. Alzheimers Res Ther. 2011 Nov; $3(6): 32$.

27 Fell J, Klaver P, Lehnertz K, Grunwald T, Schaller C, Elger CE, et al. Human memory formation is accompanied by rhinal-hippocampal coupling and decoupling. Nat Neurosci. 2001 Dec;4(12):1259-64.

28 Buffalo EA, Reber PJ, Squire LR. The human perirhinal cortex and recognition memory. Hippocampus. 1998; 8(4):330-9.

29 Convit A, de Asis J, de Leon MJ, Tarshish CY, De Santi S, Rusinek H. Atrophy of the medial occipitotemporal, inferior, and middle temporal gyri in non-demented elderly predict decline to Alzheimer's disease. Neurobiol Aging. 2000 Jan-Feb;21(1):19-26.

30 Fox NC, Warrington EK, Freeborough PA, Hartikainen P, Kennedy AM, Stevens JM, et al. Presymptomatic hippocampal atrophy in Alzheimer's disease. A longitudinal MRI study. Brain. 1996 Dec;119(Pt 6):2001-7.

31 Thompson PM, Hayashi KM, De Zubicaray GI, Janke AL, Rose SE, Semple J, et al. Mapping hippocampal and ventricular change in Alzheimer disease. Neuroimage. 2004 Aug;22(4):1754-66.

32 Mesulam MM. Neuroplasticity failure in Alzheimer's disease: bridging the gap between plaques and tangles. Neuron. 1999 Nov;24(3):521-9.

33 Fjell AM, Walhovd KB, Fennema-Notestine C, McEvoy LK, Hagler DJ, Holland D, et al.; Alzheimer's Disease Neuroimaging Initiative. CSF biomarkers in prediction of cerebral and clinical change in mild cognitive impairment and Alzheimer's disease. J Neurosci. 2010 Feb;30(6):2088-101.

34 Blennow K. Cerebrospinal fluid protein biomarkers for Alzheimer's disease. NeuroRx. 2004 Apr;1(2):213-25.

35 Gunning-Dixon FM, Raz N. The cognitive correlates of white matter abnormalities in normal aging: a quantitative review. Neuropsychology. 2000 Apr;14(2):224-32.

36 Jonsson M, Zetterberg H, van Straaten E, Lind K, Syversen S, Edman A, et al. Cerebrospinal fluid biomarkers of white matter lesions - cross-sectional results from the LADIS study. Eur J Neurol. 2010 Mar;17(3):377-82.

37 Osborn KE, Liu D, Samuels LR, Moore EE, Cambronero FE, Acosta LM, et al. Cerebrospinal fluid $\beta$-amyloid42 and neurofilament light relate to white matter hyperintensities. Neurobiol Aging. 2018 Aug;68:18-25.

38 Tang Y, Nyengaard JR, Pakkenberg B, Gundersen HJ. Age-induced white matter changes in the human brain: a stereological investigation. Neurobiol Aging. 1997 Nov-Dec;18(6):609-15.

39 Small SA, Stern Y, Tang M, Mayeux R. Selective decline in memory function among healthy elderly. Neurology. 1999 Apr;52(7):1392-6.

40 Charlton RA, Barrick TR, McIntyre DJ, Shen Y, O'Sullivan M, Howe FA, et al. White matter damage on diffusion tensor imaging correlates with age-related cognitive decline. Neurology. 2006 Jan;66(2):217-22.

41 Ziegler DA, Piguet O, Salat DH, Prince K, Connally E, Corkin S. Cognition in healthy aging is related to regional white matter integrity, but not cortical thickness. Neurobiol Aging. 2010 Nov;31(11):1912-26.

42 Menke RA, Gray E, Lu CH, Kuhle J, Talbot K, Malaspina A, et al. CSF neurofilament light chain reflects corticospinal tract degeneration in ALS. Ann Clin Transl Neurol. 2015 Jul;2(7):748-55.

43 Skillbäck T, Farahmand B, Bartlett JW, Rosén C, Mattsson N, Nägga K, et al. CSF neurofilament light differs in neurodegenerative diseases and predicts severity and survival. Neurology. 2014 Nov;83(21):1945-53.

44 Hedden T, Mormino EC, Amariglio RE, Younger AP, Schultz AP, Becker JA, et al. Cognitive profile of amyloid burden and white matter hyperintensities in cognitively normal older adults. J Neurosci. 2012 Nov;32(46): $16233-42$

45 Wirth M, Villeneuve S, Haase CM, Madison CM, Oh H, Landau SM, et al. Associations between Alzheimer disease biomarkers, neurodegeneration, and cognition in cognitively normal older people. JAMA Neurol. 2013 Dec; 70(12):1512-9.

46 Wardlaw JM, Valdés Hernández MC, Muñoz-Maniega S. What are white matter hyperintensities made of? Relevance to vascular cognitive impairment. J Am Heart Assoc. 2015 Jun;4(6):001140.

47 Gattringer T, Pinter D, Enzinger C, Seifert-Held T, Kneihsl M, Fandler S, et al. Serum neurofilament light is sensitive to active cerebral small vessel disease. Neurology. 2017 Nov;89(20):2108-14.

48 Ivanoiu A, Sindic CJ. Cerebrospinal fluid TAU protein and amyloid beta42 in mild cognitive impairment: prediction of progression to Alzheimer's disease and correlation with the neuropsychological examination. Neurocase. 2005 Feb;11(1):32-9.

49 Schoonenboom SN, Visser PJ, Mulder C, Lindeboom J, Van Elk EJ, Van Kamp GJ, et al. Biomarker profiles and their relation to clinical variables in mild cognitive impairment. Neurocase. 2005 Feb;11(1):8-13. 
50 Vemuri P, Wiste HJ, Weigand SD, Shaw LM, Trojanowski JQ, Weiner MW, et al.; Alzheimer's Disease Neuroimaging Initiative. MRI and CSF biomarkers in normal, MCI, and AD subjects: diagnostic discrimination and cognitive correlations. Neurology. 2009 Jul;73(4):287-93.

51 Negash S, Bennett DA, Wilson RS, Schneider JA, Arnold SE. Cognition and neuropathology in aging: multidimensional perspectives from the Rush Religious Orders Study and Rush Memory And Aging Project. Curr Alzheimer Res. 2011 Jun;8(4):336-40.

52 Han SD, Gruhl J, Beckett L, Dodge HH, Stricker NH, Farias S, et al.; Alzheimer's Disease Neuroimaging Initiative. Beta amyloid, tau, neuroimaging, and cognition: sequence modeling of biomarkers for Alzheimer's Disease. Brain Imaging Behav. 2012 Dec;6(4):610-20.

53 Ingelsson M, Fukumoto H, Newell KL, Growdon JH, Hedley-Whyte ET, Frosch MP, et al. Early Abeta accumulation and progressive synaptic loss, gliosis, and tangle formation in AD brain. Neurology. 2004 Mar;62(6): 925-31.

54 Gunning-Dixon FM, Raz N. Neuroanatomical correlates of selected executive functions in middle-aged and older adults: a prospective MRI study. Neuropsychologia. 2003;41(14):1929-41.

55 Fine EM, Kramer JH, Lui LY, Yaffe K; Study of Osteoporotic Fractures (SOF) Research Group. Normative data in women aged 85 and older: verbal fluency, digit span, and the CVLT-II short form. Clin Neuropsychol. 2012; 26(1):18-30.

56 Gallassi R, Sambati L, Stanzani Maserati M, Poda R, Oppi F, De Matteis M, et al. Simple verbal analogies test: normative data on a short task exploring abstract thinking. Aging Clin Exp Res. 2014 Feb;26(1):67-71.

57 Oosterhuis HE, van der Ark LA, Sijtsma K. Sample size requirements for traditional and regression-based norms. Assessment. 2016 Apr;23(2):191-202. 